\title{
Résoudre des structures cristallographiques inconnues avec un microscope électronique en transmission
}

Holger Klein (holger.klein@neel.cnrs.fr)

Institut Néel, CNRS/UJF UPR2940, 25 rue des Martyrs, BP 166, 38042 Grenoble Cedex 9

\section{Lorsqu'on ambitionne}

de comprendre et d'améliorer les propriétés des matériaux, la connaissance précise de l'organisation de la matière à l'échelle atomique est indispensable. Ainsi, depuis 100 ans, la diffraction des rayons $X$ est utilisée pour la détermination des structures. Cependant, avec l'apparition des matériaux nanométriques, cette technique commence à se heurter à ses propres limites.

\section{Depuis quelques années,}

la microscopie électronique en transmission relève le défi de la résolution de structure dans les matériaux

\section{nanométriques grâce}

à l'invention de la diffraction

électronique en précession.

Nous montrons dans

cet article les possibilités

de cette nouvelle technique.

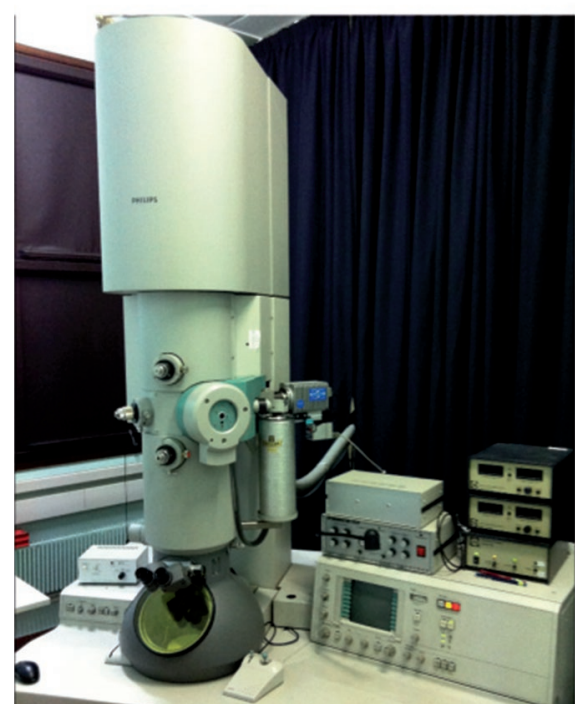

Les matériaux qui nous entourent possèdent des propriétés qui leur sont propres (dureté, isolation thermique, conductivité, combustibilité...), et nous cherchons depuis toujours à les utiliser de façon à tirer le meilleur profit de ces propriétés. Au-delà des matériaux naturels nous avons aussi appris à en confectionner de nouveaux, toujours en quête de propriétés bien particulières pour des applications spécifiques. Ainsi, le chercheur moderne en science des matériaux peut mélanger des éléments chimiques et les faire réagir afin d'obtenir un nouveau matériau. Mais plus encore que la composition chimique (que le chercheur maîtrise plus ou moins selon que l'association des éléments s'effectue plus ou moins facilement), c'est souvent l'arrangement des atomes qui détermine les propriétés du composé. Dans une grande partie des matériaux que nous utilisons (métaux, alliages, céramiques, semi-conducteurs...) cet arrangement est ordonné, on dit qu'il est " cristallin ": une unité structurale qui peut contenir seulement quelques atomes, mais parfois aussi des centaines voire des milliers d'atomes, est répétée de façon régulière dans l'espace. Cet arrangement est difficile à prévoir ; la détermination de la structure cristalline d'un matériau est un prérequis essentiel pour la compréhension de ses propriétés physiques.

Dans cet article, nous présentons la diffraction électronique en précession, technique de détermination de structures cristallines particulièrement adaptée aux matériaux nanométriques, qui prennent de plus en plus d'importance en science et technologie.
Le microscope électronique en transmission de I’Institut Néel, Grenoble.

\section{Au début de la détermination des structures : les rayons $X$}

Après la découverte des rayons X par Roentgen en 1895, W.H. Bragg et W.L. Bragg ont utilisé ces ondes à partir de 1912 afin de réaliser des expériences de diffraction sur des cristaux et de déterminer les positions atomiques dans ces structures ordonnées. La cristallographie aux rayons $\mathrm{X}$ a ensuite connu un énorme succès pour élucider les structures des minéraux, des métaux et intermétalliques ainsi que de molécules biologiques, allant de petites molécules à des protéines et même jusqu'à la double hélice de l'ADN. Aujourd'hui, on connaît plus de 100000 structures cristallines grâce à la diffraction des rayons $\mathrm{X}$.

Les techniques de diffraction des rayons $\mathrm{X}$ ont été étudiées depuis une centaine d'années et sont donc bien connues aujourd'hui. Néanmoins, un cristal qui doit être étudié par diffraction des 


\section{\>}

rayons $\mathrm{X}$ doit avoir une taille minimum. Typiquement, pour des expériences de diffraction réalisées en laboratoire il est nécessaire de disposer d'un cristal d'environ $50 \mu \mathrm{m}$ de diamètre. Pour celles réalisées dans un synchrotron, une de ces grandes installations qui constituent les plus brillantes sources de rayons $\mathrm{X}$, la taille nécessaire n'est plus que de quelques microns. Si la taille du cristal descend en dessous de cette limite, les intensités des rayons X diffractés par un seul cristal deviennent tellement faibles qu'il est impossible de les utiliser pour la détermination de sa structure.

Or, bien que déjà très petits, ces cristaux sont encore énormes par rapport aux nanoparticules. Ces dernières peuvent avoir des diamètres de quelques dizaines de nanomètres (voire moins !), soit cent fois plus petits que les plus petits cristaux que l'on puisse étudier au synchrotron. Et un diamètre cent fois plus petit signifie un volume un million de fois plus petit, ce qui se traduit par des intensités diffractées également un million de fois plus faibles. Même les progrès techniques auxquels on peut s'attendre dans l'avenir ne permettront probablement pas de résoudre la structure d'une nanoparticule unique par diffraction des rayons $\mathrm{X}$.

Si l'on ne dispose que de cristaux dont les tailles sont inférieures aux limites mentionnées ci-dessus, on peut toutefois réaliser des expériences de diffraction de rayons $\mathrm{X}$ sur une poudre constituée d'un très grand nombre de ces petits cristaux. Dans ce cas, on obtient l'information de diffraction sur l'ensemble des particules contenues dans la poudre, c'est-à-dire la moyenne sur toutes les particules. Par contre, on perd l'information sur l'orientation et l'identité de chaque particule individuelle. Si la poudre est monophasée, c'est-à-dire si elle ne contient qu'une seule structure cristalline et que cette structure inconnue n'est pas trop complexe, ces informations moyennes suffisent pour la détermination de la structure et, en effet, un grand nombre de structures inconnues ont ainsi pu être résolues.

Mais quid des poudres de cristaux complexes ou qui contiennent plusieurs phases inconnues? Les rayons $\mathrm{X}$ seuls ne suffisent plus, il faut alors envisager l'utilisation d'une autre sonde pour les étudier. Ces dernières années, il a été montré que cette autre sonde peut être un faisceau d'électrons dans un microscope électronique en transmission (encadré 1).

\section{La diffraction sur des nanoparticules : les électrons prennent la relève}

Dans un microscope électronique en transmission (MET), les électrons sont accélérés par une haute tension et la longueur d'onde associée vaut quelques centièmes d'ångström, c'est-à-dire environ un centième de la distance qui sépare les atomes dans un cristal. Les électrons vus comme des ondes sont donc aptes à être diffractés par l'arrangement périodique des atomes dans un cristal (fig. 1).

Contrairement aux rayons $\mathrm{X}$, les électrons portent une charge électrique. Par conséquent, ils interagissent avec les charges des noyaux et des électrons du cristal. Cette interaction étant beaucoup plus forte que celle des rayons $\mathrm{X}$ avec la matière, il est possible de faire des expériences de diffraction électronique avec des cristaux beaucoup plus petits. D'autre part, la charge électrique des électrons permet d'utiliser des lentilles électromagnétiques afin de focaliser un faisceau d'électrons sur un cristal nanométrique.

Les électrons sont donc particulièrement adaptés pour étudier la structure des nanocristaux.

Cependant, et bien qu'ils aient été utilisés pour les expériences de diffraction dans un microscope électronique en transmission depuis 1933, les électrons

\section{Le microscope électronique en transmission (MET)}

encadré 1

Le MET a été inventé dans les années 1930, afin d’obtenir des images avec une résolution supérieure à celle d'un microscope optique, qui est limitée par la longueur d'onde de la lumière. Les électrons sont généralement vus comme des particules, mais d'après I'hypothèse de L. de Broglie on peut associer une onde à chaque particule en mouvement. Il est donc possible de faire des expériences de diffraction avec des électrons. Dans le MET, ceux-ci sont accélérés par une haute tension comprise en général entre $80 \mathrm{kV}$ et $400 \mathrm{kV}$. Dans ce cas, les électrons ont des longueurs d'onde comprises entre 0,042 $\AA$ et 0,016

Les lentilles utilisées dans un MET sont des bobines parcourues par un courant électrique. Le champ magnétique créé par ces bobines dévie les électrons et on peut ainsi former des images agrandies. Les MET modernes permettent d'obtenir des images avec une résolution atomique et de la diffraction sur une même particule nanométrique.

La figure E1 montre un exemple de cliché de diffraction électronique obtenu sur un monocristal. Les réflexions forment un réseau bidimensionnel, qui correspond à un plan du réseau réciproque. Les distances entre les réflexions dans une rangée et les angles entre les rangées permettent de déterminer les paramètres de maille du cristal. Les extinctions systématiques de certaines réflexions donnent des informations sur la symétrie du cristal.

Comme l'interaction des électrons avec la matière est très forte, l'épaisseur de l'échantillon doit être très petite sans quoi le faisceau ne pourrait pas traverser l'échantillon. Cette épaisseur doit ainsi mesurer entre 5 et $100 \mathrm{~nm}$, ce qui nécessite des méthodes de préparation spécifiques. Malgré cette extrême finesse des échantillons, une grande partie des électrons subit non seulement une mais plusieurs diffractions avant d'en ressortir. Cette diffraction multiple rend l'interprétation des intensités diffractées très difficile.

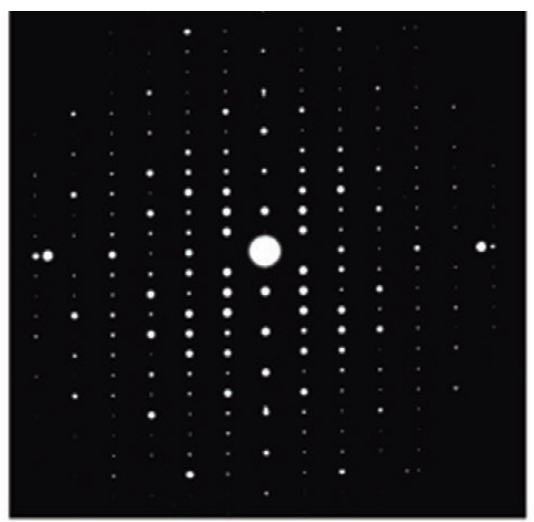

E1. Cliché de diffraction en axe de zone d'un cristal orthorhombique d'une phase intermétallique Al-Pd-Mn. Les rangées centrales horizontale et verticale ne montrent qu'une réflexion sur deux, les autres étant éteintes à cause des éléments de symétrie du cristal (axes hélicoïdaux). 
ont pendant longtemps été inadaptés à la résolution de structures cristallines inconnues, justement à cause de la forte interaction des électrons avec la matière. Cette forte interaction, qui constitue l'avantage des électrons par rapport aux rayons $\mathrm{X}$, est en même temps un inconvénient. En fait, l'interaction entre les électrons du faisceau et la matière est tellement forte que, malgré la faible épaisseur des échantillons, un électron diffracté dans le cristal a toutes les chances de l'être une deuxième voire une troisième fois avant d'avoir traversé complètement l'échantillon. Ainsi, l'approximation dite cinématique, couramment utilisée en diffraction des rayons $\mathrm{X}$, et qui suppose qu'une particule n'est diffractée qu'une seule fois à l'intérieur du cristal, n'est plus valide pour les électrons.

Or, dans la procédure de résolution d'une structure il faut calculer les facteurs de structure cristalline à partir des intensités mesurées. Ce sont ces facteurs de structure qui contiennent l'information relative aux positions atomiques dans le cristal (voir encadré 2). Malheureusement ce calcul, simple dans l'approximation cinématique, devient très complexe en présence de diffractions multiples comme c'est le cas pour les électrons. En particulier, le calcul nécessite de connaitre la structure que l'on veut déterminer, ce qui n'est bien sûr jamais le cas lorsqu'on étudie un cristal inconnu.

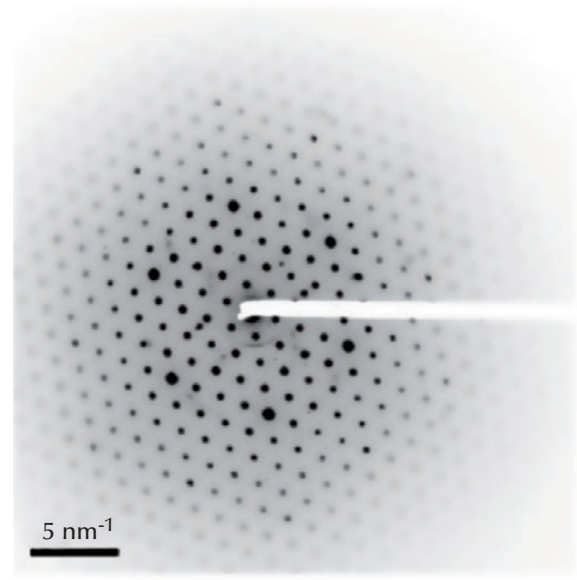

1. Un cliché de diffraction électronique de l'axe de zone [111] de la phase cubique $\mathrm{Mn}_{2} \mathrm{O}_{3}$, obtenu en diffraction « classique ». Toutes les réflexions présentent des intensités similaires.

\section{Comment résoudre une structure cristalline}

Lors d'une expérience de diffraction sur une structure cristalline, chaque atome contribue aux intensités diffractées en fonction de sa nature et de sa position dans la maille élémentaire. Chaque atome diffuse l'onde incidente, et toutes ces ondes diffusées interfèrent pour donner les ondes diffractées. L'amplitude de chaque onde diffractée, que l'on appelle "facteur de structure ", contient donc les contributions de tous les atomes. Les facteurs de structure (décrits par leurs modules et leurs phases) sont les composantes de Fourier du pouvoir de diffusion du cristal (densité électronique pour les rayons $X$, potentiel électrostatique du cristal pour les électrons). Afin de résoudre une structure, on doit donc déterminer un grand nombre de facteurs de structures. Lors de la diffraction, l'information sur les phases des facteurs de structure est perdue, mais l'intensité diffractée contient l'information sur leurs modules. Un grand nombre de méthodes et de logiciels ont été développés afin de retrouver les phases, le travail de l'expérimentateur consistant à déterminer les modules des facteurs de structure. Dans le cas de la diffraction des rayons $X$, cela est très simple. Dans l'approximation cinématique où un rayon $\mathrm{X}$ n'est diffracté qu'une seule fois dans le cristal, l'intensité mesurée est égale au carré du module du facteur de structure. Dans le cas de la diffraction électronique, cette approximation n'est pas valable et la relation entre l'intensité et le facteur de structure est complexe. C'est le mérite de la diffraction électronique en précession de fournir des intensités diffractées qui s'approchent d'une relation simple avec les modules des facteurs de structure. On peut ainsi obtenir des valeurs de ces derniers qui sont suffisamment précises pour résoudre une structure.

Expérimentalement, la première étape dans la résolution d'une structure est la réalisation d'une expérience de diffraction. Dans le cas de la cristallographie aux électrons, cette expérience se fait dans un MET. L'échantillon est orienté avec une direction cristallographique particulière parallèle au faisceau incident (appelée " axe de zone »), et le cliché de diffraction est enregistré. Ensuite, l'échantillon est tourné de façon à obtenir le plus grand nombre d'axes de zone possibles, dont les clichés de diffraction sont enregistrés (fig. E2). Grâce à l'ensemble des clichés et connaissant les angles entre les axes de zone, on peut ainsi reconstruire la forme et la taille de la maille élémentaire du cristal ainsi que ses symétries.

Enfin, les intensités diffractées sont extraites des clichés de diffraction et les modules des facteurs de structure sont calculés. Ces données sont introduites dans un des nombreux logiciels de la cristallographie classique, afin d'obtenir un premier modèle de la structure.

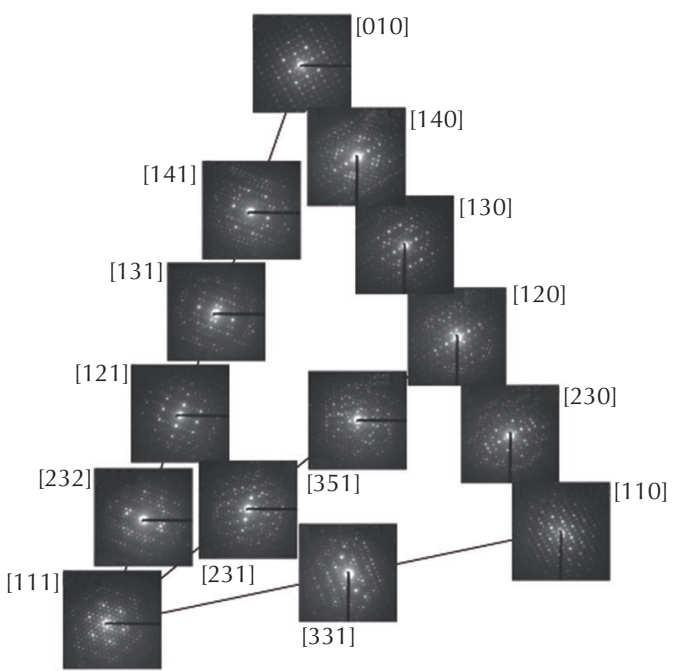

E2. Clichés de diffraction des électrons par un cristal de $\mathrm{Mn}_{2} \mathrm{O}_{3}$, pour plusieurs orientations (axes de zone). Les clichés ont été obtenus en tournant le cristal autour d'un axe cristallographique. Les indices des axes de zone sont donnés entre crochets sur chaque cliché de diffraction. 


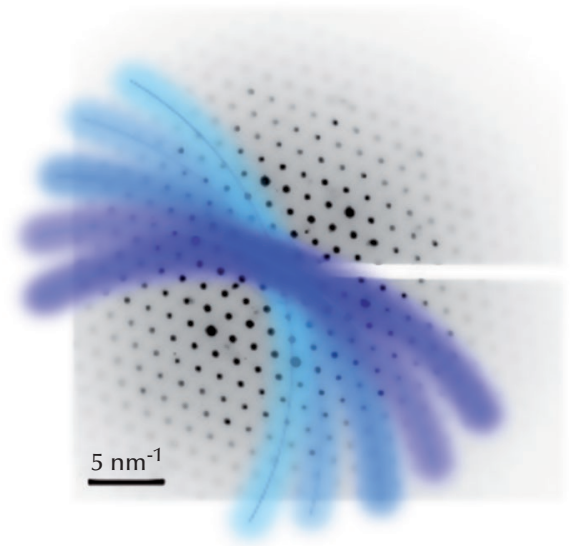

2. Illustration de l'effet de la précession sur le cliché de diffraction de la figure 1. À un moment donné, seules les réflexions recouvertes par l'un des anneaux colorés sont en condition de diffraction. La rotation du faisceau incident fait tourner l'anneau des réflexions. Un temps de pose long par rapport au temps nécessaire au faisceau pour effectuer un tour complet permet d'enregistrer toutes les réflexions du plan.

\section{\>}

\section{La diffraction électronique en précession (PED) : une manière de contourner le problème}

La technique de diffraction électronique en précession (PED), introduite en 1994 par Vincent et Midgley [1], réduit considérablement les problèmes liés à la diffraction multiple. Alors que le cristal est en orientation d'axe de zone avec l'axe optique du microscope, le faisceau incident est légèrement incliné par rapport à cet axe. Par conséquent, le cliché de diffraction ne comporte pas comme en diffraction " classique " tout un plan du réseau réciproque (fig. 1), mais seulement un anneau de réflexions. Dans la figure 2, cet anneau est représenté par l'arc de cercle bleu superposé au cliché en axe de zone.

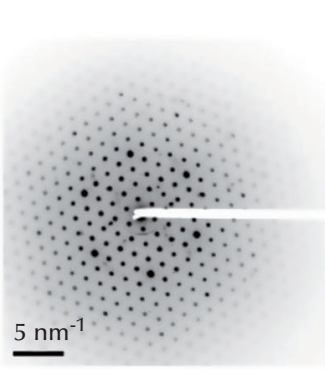

$\mathrm{a}$

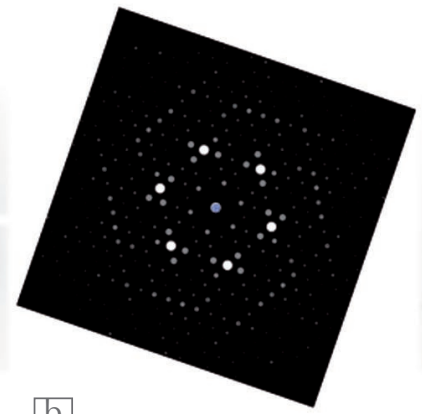

b
Il y a donc beaucoup moins de réflexions excitées en même temps, ce qui réduit les possibilités de diffraction multiple.

Le faisceau incident ainsi incliné est mis en mouvement autour de l'axe optique, et décrit une surface conique avec un point fixe sur l'échantillon. Ainsi, l'anneau des réflexions excitées tourne autour du faisceau transmis (anneaux bleus/violets dans la figure 2). Toutes les réflexions du plan sont excitées successivement. Comme le temps pour que le faisceau effectue une précession est très court comparé au temps d'exposition, on enregistre un cliché contenant toutes les réflexions du plan (fig. 3c).

La comparaison entre un cliché de diffraction "classique " (fig. 3a) et un cliché obtenu par PED (fig. 3c) avec le même cliché calculé en théorie cinématique (fig. 3b), montre clairement que le cliché PED ressemble beaucoup plus à ce qu'on attend en théorie cinématique.

Les intensités mesurées ainsi permettent ensuite de déterminer les valeurs des facteurs de structure, au moins de façon approximative [2]. Une détermination précise des facteurs de structure n'est toutefois pas possible car, même si la PED réduit la diffraction multiple, elle ne l'élimine pas complètement. Par conséquent, la communauté des cristallographes a eu de grands doutes quant aux possibilités réelles de résoudre des structures inconnues par PED, et cela même après que les premières solutions de structures cristallines aient été publiées. Ce n'est que récemment qu'il a été montré que la diminution de la diffraction multiple par la précession est suffisante pour que les facteurs de structure extraits des intensités diffractées en PED permettent la résolution des structures cristallines de façon fiable.
3. Comparaison entre un cliché de diffraction « classique » (a) et un cliché obtenu par précession (c) avec les intensités calculées en théorie cinématique (b), de l'axe de zone [111] de la phase cubique $\mathrm{Mn}_{2} \mathrm{O}_{3}$. Les intensités obtenues en précession sont beaucoup plus proches des valeurs théoriques, sans toutefois être identiques.

\section{Résoudre une structure cristalline par diffraction électronique en précession}

La diffraction " classique " des électrons permet de déterminer les paramètres de maille du cristal, ainsi que ses symétries. Une fois ces informations obtenues, le travail du microscopiste cristallographe consiste à donner au cristal une orientation cristallographique particulière (axe de zone) dans le MET et à enregistrer les intensités diffractées en PED. Il cherchera ensuite d'autres orientations du cristal afin d'enregistrer un maximum d'intensités diffractées. En utilisant des logiciels standard de la cristallographie, on peut ensuite résoudre des structures telles que celle montrée en figure 4. Si l'on compare ce modèle obtenu par diffraction électronique avec la véritable structure du cristal, on constate qu'il est essentiellement correct. Les positions atomiques calculées sont correctes et assez précises [3]. Cependant, on constate également dans cet exemple les limites de cette méthode : la position d'un atome d'oxygène manque dans ce modèle, et certaines positions d'oxygène ne sont pas occupées à 100\%. La précision des données de diffraction électronique utilisées n'est pas suffisante pour la détermination de ces détails.

Dans des travaux récents, nous avons étudié systématiquement l'influence de plusieurs paramètres sur le succès de la résolution d'une structure cristallographique à partir des données de PED [4]. Ces paramètres sont, entre autres : l'angle de précession, le nombre d'axes de zone utilisés et la résolution maximale des données.

Le nombre de réflexions mesurées semble être le paramètre prépondérant. Pour améliorer les chances de résoudre la structure, nous avons besoin d'un grand nombre de réflexions. Ce nombre dépend du nombre d'axes de zone accessibles et de la limite de résolution qui peut être atteinte dans l'espace réciproque. De manière surprenante, l'efficacité de la cristallographie aux électrons n'est pas influencée par la précision de la mesure des intensités des réflexions ou par celle de l'extraction des facteurs de structure à partir de ces intensités, à condition que la qualité des données soit telle que les réflexions " intenses » soient effectivement intenses et que les réflexions " peu intenses » soient effectivement peu intenses. 

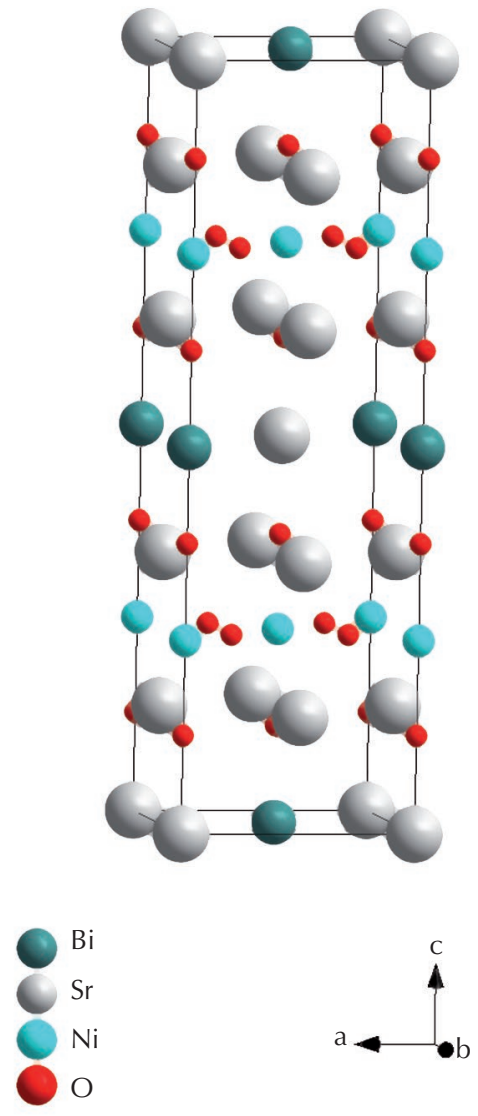

4. Exemple d'une structure résolue par cristallographie aux électrons : l'oxyde quaternaire $\mathrm{Sr}_{5} \mathrm{BiNi}_{2} \mathrm{O}_{9,6}$. La connaissance de ce modèle de structure a, par la suite, permis de faire un affinement des positions avec des données de diffraction de rayons $X$ sur poudre. Cet affinement a montré que les positions initialement trouvées par cristallographie aux électrons étaient situées à environ $0,1 \AA$ des positions affinées. Une position d'oxygène dans le plan de base de la maille n’a pas été trouvée.
Pour aboutir à cette conclusion, nous avons utilisé les données de PED expérimentales qui avaient déjà été traitées pour la résolution des structures. Nous avons classé les réflexions en seulement trois catégories selon leurs intensités respectives : fortes, moyennes ou faibles. Dans chaque classe, nous avons attribué la même intensité (l'intensité moyenne de la classe) à toutes les réflexions. Nous avons "détérioré » les données de cette façon pour plusieurs structures cristallines différentes, simples puis de plus en plus complexes, montrant une basse ou une haute symétrie, et des cristaux contenant des éléments lourds ou légers, ou les deux [5]. Les structures déduites de ces données détériorées volontairement sont (presque) aussi correctes que celles obtenues précédemment à partir des données mesurées. On obtient toujours la même précision pour les positions des cations ; seule la précision des positions des atomes légers est parfois légèrement réduite. Les effets de diffraction multiple, principal obstacle à une détermination précise des facteurs de structure en diffraction des électrons, ne sont donc plus un obstacle à la résolution de la structure, une fois qu'ils ont été suffisamment réduits par la précession du faisceau d'électrons.

\section{Références}

1 R. Vincent et P.A. Midgley, Double conical beamrocking system for measurement of integrated electron diffraction intensities Ultramicroscopy 53 (1994) 271. 2• H. Klein, Z. Kristallogr. 228 (2013) 35.

\section{Conclusion}

La cristallographie aux rayons $\mathrm{X}$ a permis la résolution d'un très grand nombre de structures cristallines. Notre compréhension des matériaux en a été fortement améliorée. Les avancées technologiques des dernières décennies n'auraient pas été possibles sans cette compréhension. À l'heure où la diffraction des rayons $\mathrm{X}$ atteint parfois ses limites, nous avons montré que la cristallographie aux électrons est prête à prendre la relève. Même si les données obtenues avec cette dernière ne sont pas aussi précises que celles de la diffraction des rayons $\mathrm{X}$, leur qualité est suffisante pour la résolution de structures, même complexes. Il ne s'agit toutefois pas de remplacer la cristallographie aux rayons $\mathrm{X}$ par la diffraction des électrons, mais plutôt de disposer d'une méthode tantôt alternative tantôt complémentaire, et surtout très adaptée dans certains cas où les rayons $\mathrm{X}$ se révèlent impuissants.

La combinaison des méthodes cristallographiques basées sur les rayons $\mathrm{X}$, les électrons et - pourquoi pas ? - les neutrons, promet de décupler l'étendue du champ d'action et la puissance de la cristallographie.

3• M. Novitskaya et al., J. Solid State Chem. 184 (2011) 3262.

4• H. Klein, Acta Cryst. A67 (2011) 303.

5• H. Klein et J. David, Acta Cryst. A67 (2011) 297. 